\title{
Modified Design of Novel Variable-Focus Lens for VHFGW
}

\author{
R. Clive Woods \\ 102A Department of Electrical and Computer Engineering, South Campus Drive, \\ Louisiana State University, Baton Rouge, LA 70803-5901, U.S.A.. \\ (225) 578 5243,Fax(225) 578 5200, cwoods@lsu.edu
}

\begin{abstract}
The present author previously published a paper at STAIF 2006 detailing the design of a novel variablefocus lens for use with very-high frequency gravitational waves (VHFGW, typically around $3 \mathrm{GHz}$ ). Such a lens would be invaluable in the design of advanced GW optics for communications applications since focusing would be achievable electrically with no moving parts. The design was based upon the published calculations of Li and Torr (in 1992 and 1993) claiming to show that GWs propagate inside superconductors with a phase velocity reduction by a factor $n \sim 300 \times$ and a corresponding wavenumber increase. Successful demonstration of this lens would also confirm the controversial Li and Torr result. Type II superconductors do not completely expel large magnetic fields, but instead allow vortices of magnetic flux to channel the magnetic field through the material. Within these vortices, the superconductor is magnetically quenched and so has properties similar or identical to those of non-superconductors. Varying the applied magnetic field varies the proportion of material quenched and superconducting. For GW wavelengths significantly larger than the typical vortex separation, the GW propagation through a type II superconductor is therefore dependent upon the applied magnetic field. Since a conventional optical lens may be regarded as a position-dependent phase shifter, and the VHFGW phase-shift depends upon the applied magnetic field, the design of a VHFGW lens therefore reduces to producing a suitable applied magnetic field variation that gives a technologically-useful spatial variation of phase shift. In this paper, a modified method of producing the required magnetic field is introduced, as well as a more detailed discussion of the VHFGW propagation through the superconducting material with an applied field.
\end{abstract}

Keywords: Gravitational waves, HFGW, high-frequency gravitational waves, lens, magnetic field, magnetic vortex, optical blooming, optical coating, superconductors, waveguiding, wave impedance, very high-frequency gravitational waves, VHFGW.

PACS: 85.25.Am.

\section{INTRODUCTION}

Gravitational waves are now widely recognized as a highly important emerging technology for future communications and propulsion. The initial theoretical work of Einstein (1916), Forward and Miller (1966), and Romero and Dehnen (1981) laid the foundations supporting subsequent experimentation. Confirmed observations of very-low-frequency gravitational waves of astronomical origin eliminated any remaining doubts concerning their existence and nature (Taylor, 1994). Subsequent development has included the large-scale LISA, LIGO, Virgo, and other prototype projects for low-frequency gravitational wave detection. More recently, the properties of highfrequency gravitational waves (HFGWs, i.e., those containing components at frequencies between $100 \mathrm{kHz}$ and $100 \mathrm{MHz}$ ) and very-high-frequency gravitational waves (VHFGWs, i.e., those containing components at frequencies between $100 \mathrm{MHz}$ and $100 \mathrm{GHz}$ ) (Douglass and Braginsky, 1979) have been investigated theoretically, together with suggested methods of generating and detecting them in a laboratory (Woods and Baker, 2005) and of manipulating them for technological purposes (Woods, 2005).

$\mathrm{Li}$ and Torr (1992) and Torr and Li (1993) have published calculations of the propagation behavior of gravitational waves inside superconductors. They claimed that the phase velocity of gravitational waves in any superconducting material would be $\sim 10^{6} \mathrm{~m} \mathrm{~s}^{-1}$, that is $\sim 300$ times less than the phase velocity of gravitational waves in other materials. This is generally assumed to be equal to the speed of light in a vacuum $\left(c=3 \times 10^{8} \mathrm{~m} \mathrm{~s}^{-1}\right)$. Therefore, using the language of conventional geometric optics, what is claimed is that a superconductor has a "GW refractive index" $n_{g} \approx 300$. This also causes the wavenumber to increase by a corresponding factor $n_{g} \approx 300$ times in superconducting 
materials.

This result has major consequences for the design of future instruments to generate and detect gravitational waves, which may need to be able to focus, refract, reflect, and otherwise manipulate gravitational waves for efficient coupling to detectors, transmitters, generators, resonant chambers, and other sensors. Woods (2006a) illustrated some of these consequences exploiting the Li and Torr result by outlining the design of a novel type of HFGW lens design using a magnetic field to adjust the focal length in an infinitely-variable manner. The object of the present paper is to describe a modification to this device that may be easier to realise in practice.

\section{BASIC PRINCIPLES OF VHFGW LENS}

Woods (2006b) showed that when travelling through a type-II superconductor, VHFGW interact with the magnetic vortex structure in such a way that the propagation constant is renormalized. As a result; the phase shift of a VHFGW after travelling through a type-II superconductor of thickness $b$ is approximately given by

$$
\begin{aligned}
\phi & \approx \omega\left(\frac{n_{g}}{c}-\frac{\sqrt{3} \pi^{2} B c}{n_{g} \omega^{2} \Phi_{\mathrm{o}}}\right) b \\
& =\left(\frac{n_{g} \omega}{c}\right) b-\left(\frac{c}{n_{g} \omega}\right)\left(\frac{\sqrt{3} \pi^{2}}{\Phi_{\mathrm{o}}}\right) B b,
\end{aligned}
$$

where the magnetic flux through each vortex is $\Phi_{\mathrm{o}}$. This gives $\phi=n \omega b / c$ for $B=0$, as expected, and the phase $\phi$ can be varied continuously by varying the magnetic field $B$.

For a uniform value of applied magnetic field $B(r)$, all that is produced is a variable GW phase shifter. Lens action results when a non-uniform $B(r)$ is used. Suppose that the applied field $B(r)$ has cylindrical symmetry. Then in conventional geometric ray optics, if the phase shift varies according to

$$
\phi=\phi_{0}-\pi r^{2} /\left(\lambda_{0} f\right),
$$

where $\lambda_{\mathrm{o}}$ is the wavelength in free space and $\phi_{\mathrm{o}}$ is a constant, then this non-uniform phase shift will act as a lens of focal length $f$ for small values of phase shift and small values of $r$ (in deriving Eq. (3) it is assumed that $r / f$ is a small angle). Hence, equating the phase shift formulae in Eqs. (2) and (3), the required variation of applied magnetic field for producing a lens of focal length $f$ is given by

$$
B(r)=B(0)+\frac{n_{g} r^{2} \omega^{2} \Phi_{\mathrm{o}}}{2 \sqrt{3} \pi^{2} f c^{2} b},
$$

where $B(0)$ is the applied field on the cylindrical axis. In interpreting the value of focal length $f$, note that a negative focal length $(f<0$, corresponding to a diverging lens) is obtained if $B$ has its greatest value on the cylindrical axis.

In the original design of variable VHFGW lens (Woods, 2006a), the required quadratic variation of magnetic field was produced using a ring coil surrounding the superconducting disk used in the lens. In order to obtain a magnetic field that is cylindrically symmetric and also dependent quadratically on $r$, it may be more convenient to use a small coil of wire carrying a current and located on the cylindrical axis, as shown in Fig. 1. If the coil is small compared to the other dimensions in the experimental geometry, the coil may be approximated as a perfect magnetic dipole. The usual expression for the field from a perfect (small) magnetic dipole is (Cheng, 1989):

$$
\mathbf{B}=\frac{\mu_{\mathrm{o}} m}{4 \pi R^{3}}\left(\mathbf{a}_{R} 2 \cos \theta+\mathbf{a}_{\theta} \sin \theta\right)
$$

where the dipole moment is $m=I A$ for a current $I$ enclosing an area $A$, the radial distance from the dipole is $R$, the vectors $\mathbf{a}_{R}$ and $\mathbf{a}_{\theta}$ are unit-length basis vectors for the coordinates $R$ and $\theta$ respectively, and $\theta$ is the angle that $\mathbf{a}_{R}$ makes to the dipole axis (the same as the cylindrical axis in the present device). In the present application, the dipole field formula must be re-written in terms of the magnitude of the field on a thin planar plate of superconductor at radius $r$ from the cylindrical axis, since for small angles the GW phase shift is dependent upon the field total 
magnitude rather than on one resolved component. From Eq. (5), invoking the small-angle trigonometric approximations shows that for small $r$ the total field magnitude is given by:

$$
B(r)=\frac{\mu_{\mathrm{o}} m}{4 \pi s^{3}}\left[2-\frac{15}{4}\left(\frac{r}{s}\right)^{2}\right]=B(0)-\frac{15 \mu_{\mathrm{o}} m r^{2}}{16 \pi s^{5}}
$$

where $s$ is the axial distance from the dipole to the thin superconductor disk (and $s \gg b$ ). In this expression, the expansion is taken only as far as the term in $r^{2}$; there is no term in $r^{3}$ since by symmetry no odd powers of $r$ exist in the expansion. Therefore, equating Eqs. (4) and (6) gives the following expression for the GW focal length $f$, provided $f \gg b$ :

$$
f=-\frac{8 n_{g} \omega^{2} \Phi_{0} s^{5}}{15 \sqrt{3} \pi \mu_{0} c^{2} b m}
$$

The focal length is now inversely proportional to the independent and easily variable parameter $m$, the magnetic dipole moment. A short focal length is obtained by using a magnetic coil close to the superconductor, a thick slab of superconductor, and a large magnetic dipole moment. In this expression, the focal length will always be negative, giving a diverging lens for all parameter values, as in the case of the previous design using a large coil wrapped around the superconductor slab (Woods, 2006a) but, as before in that case, a variable focal-length converging lens can easily be constructed by making one of the superconductor faces convex rather than planar as assumed here for simplicity. The usual formulae for combining optical lenses will apply.

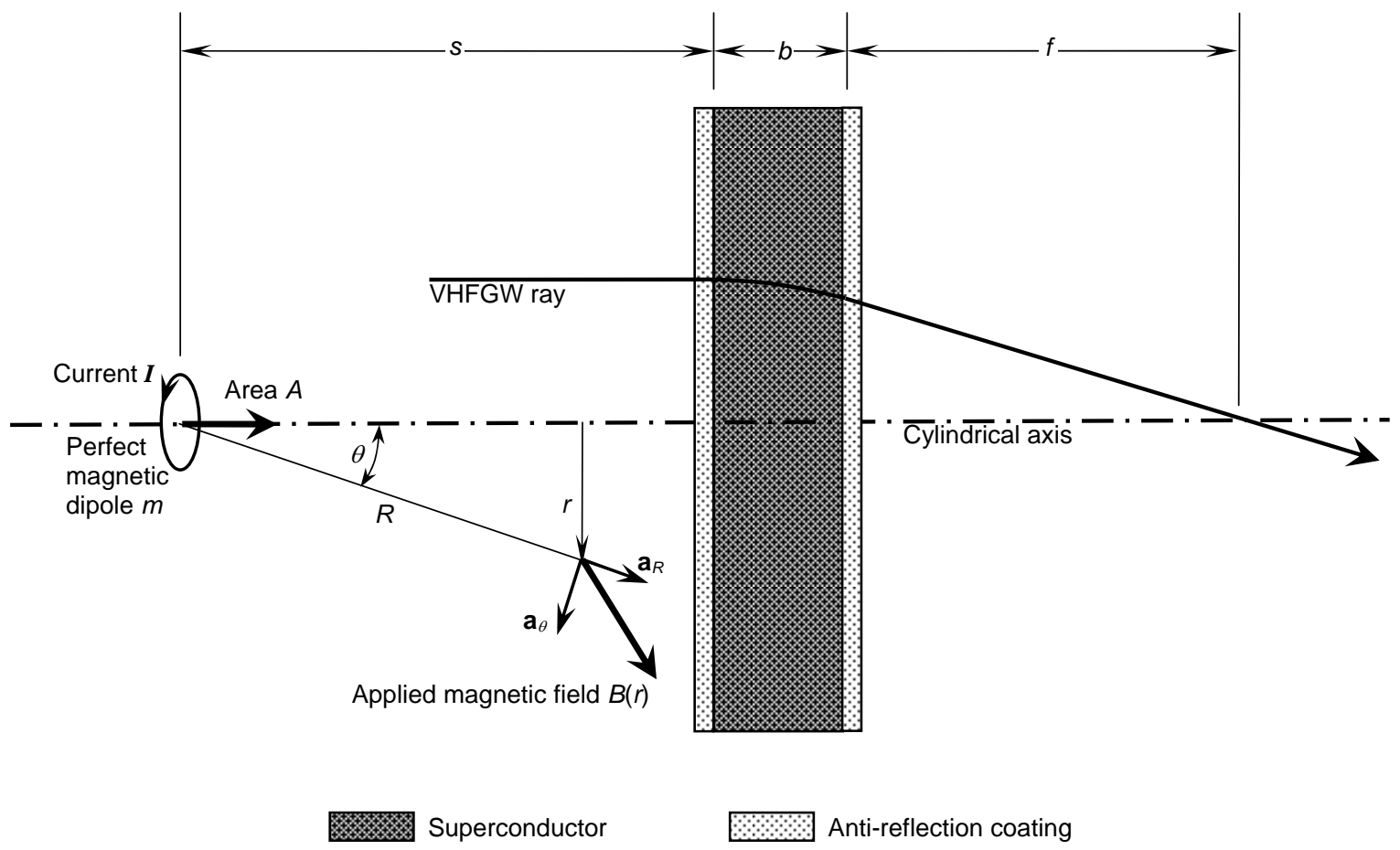

FIGURE 1. Basic VHFGW Variable-Focus Lens Using Magnetic Dipole for Producing Required Magnetic Field.

As in the previous case, there will undoubtedly be large Fresnel reflection effects from the front and back surfaces of the superconductor slab producing the focusing effect. These will occur whether they are planar or curved (for the purposes of introducing some background lens capability at zero applied field). Therefore, as before, an effective anti-reflection coating is essential on both front and back surfaces, if enormous reflection losses are to be avoided. This may be produced as described by Woods (2005).

The main advantages of the present arrangement over the previous design (Woods, 2006a) are that the focussing is controlled remotely; there is no need for a large circular coil surrounding the superconductor slab; and there is no 
need to make the superconductor precisely circular as in the previous case. If remote focussing is not required, and if the cost and provision of a large circular coil are not prohibitive, and if the superconductor can be made precisely circular, then the previous arrangement may be found more suitable than the present design.

\section{PRACTICAL DESIGN OF GW VARIABLE-FOCUS LENS}

The basic VHFGW lens depicted in Fig. 1 is diverging. The most common simple requirement will be that of a variable-focus converging lens. This would be needed (for example) to focus generated VHFGW onto the sensitive aperture of a VHFGW detector (Woods and Baker, 2005), in circumstances where the value of the GW refractive index $n_{g}$ is not known precisely enough in advance so that a lens of accurate focal length cannot be designed and made beforehand. In fact there are certainly very large error limits upon the value of $n_{g}$ currently predicted by $\mathrm{Li}$ and Torr (1992), on the order of $\pm 50 \%$ or more, due to their use of linearized theoretical approximations, so this scenario is not unrealistic. To allow for this uncertainty in a practical design, one strategy will be to manufacture a converging lens (Woods, 2005) rather stronger than actually required, assuming a value of $n_{g}$ as accurately known as possible, and where the error limits on $n_{g}$ are such that even at the lowest extreme value of $n_{g}$ possible this lens will still be slightly stronger than necessary. This converging lens must then be combined with a variable-focus diverging lens (Fig. 1) designed as described above, and the variable-focus diverging lens may be adjusted until the focal length of the combination is precisely what is required.

For the coupled-electron wavefunction to be single-valued and continuous, it is well-known that the value of the magnetic flux through each vortex, $\Phi_{0}$, must be quantized in units of the fundamental flux quantum, $h /(2 e)=$ $2.08 \times 10^{-15} \mathrm{~Wb}$, where $h$ is Planck's constant and $e$ is the fundamental charge. Assuming one flux quantum per vortex, then in terms of current $I$ and magnetic dipole area $A$ the focal length in Eq. (7) becomes:

$$
f=-\frac{4 n_{g} \omega^{2} h s^{5}}{15 \sqrt{3} \pi \mu_{\mathrm{o}} c^{2} e b I A} .
$$

Suppose that VHFGW at frequency $3 \mathrm{GHz}$ must be manipulated by a lens designed according to Fig. 1 . Taking $n_{g}=$ 300 ( $\mathrm{Li}$ and Torr, 1992), and the other fundamental physical constants take their usual values. The remaining variable parameters available to the lens designer are the dipole coil current $I$, the dipole-superconductor distance $s$, the superconductor thickness $b$, and the dipole coil area $A$. Table 1 shows some realistic selected values of these parameters, corresponding to typical design decisions that might be made, and the resulting value of the focal length calculated according to Eq. (8). Also tabulated is the maximum aperture possible for each design, listed as the " $f$ /number" corresponding to the focal length. This is calculated from a maximum usable active radius arbitrarily chosen as $s / 10$, so that the small-angle approximations used in the analysis will still be accurate.

TABLE 1. Values of focal length and aperture for selected design parameters calculated according to Eq. (8).

\begin{tabular}{cccccc}
\hline $\begin{array}{c}\text { Coil current, } \\
\mathbf{I} / \mathbf{A}\end{array}$ & $\begin{array}{c}\text { Coil-superconductor } \\
\text { distance, } \mathbf{s} / \mathbf{m}\end{array}$ & $\begin{array}{c}\text { Superconductor } \\
\text { thickness, } \mathbf{b} / \mathbf{m}\end{array}$ & Coil area, $\mathbf{A} / \mathbf{m}^{2}$ & $\begin{array}{c}\text { Focal length, } \\
\boldsymbol{f} / \mathbf{m}\end{array}$ & Aperture \\
\hline 0.05 & 0.1 & 0.01 & 0.0001 & -0.038 & $f / 1.9$ \\
1 & 0.2 & 0.01 & 0.0001 & -0.061 & $f / 1.5$ \\
10 & 0.5 & 0.003 & 0.0001 & -1.99 & $f / 20$ \\
1 & 0.3 & 0.003 & 0.00005 & -3.09 & $f / 52$ \\
2 & 0.4 & 0.003 & 0.00005 & -6.52 & $f / 82$ \\
\hline
\end{tabular}

At zero coil current, the focal length is negative infinite (i.e., no diverging or converging properties), so by varying the coil current the focal length is infinitely variable between negative infinity and the focal lengths listed in Table 1. In practice, the magnetic dipole coil can be made using many turns of wire so that the current drive actually necessary will be reduced from the value shown in Table 1 by a factor equal to the number of turns in the dipole coil.

The design parameters used in Table 1 are certainly easy to obtain experimentally and the resultant focal lengths are within the range of experimental values that will be useful to the designer of instruments and detectors of VHFGW. Reasonable values of focal lengths are obtainable without using unreasonable design parameters or presaging future 
developments necessary to enable this technology. In several cases, very fast lenses (i.e., large aperture) are produced which will have particular value in the design of instruments to detect VHFGW of very small intensity, where both the lens speed and the availability of infinitely adjustable focussing are important.

\section{CONCLUSIONS}

The design of a novel diverging lens component suitable for use with VHFGWs has been modified. This design modification enables the control of the focal length to be undertaken remotely from the superconductive phasechange element, which may be advantageous in some instrument configurations. As previously, this device produces an infinitely variable VHFGW focal length, in this case adjustable by varying the current drive to a small magnetic coil or dipole that produces a non-uniform magnetic field over the face of a plane disk of type-II superconductor.

The operation of this component relies upon the result of Woods (2006b) that the phase shift of VHFGW travelling through a type-II superconductor is directly dependent upon the applied magnetic field. In turn, this relies upon a result of Li and Torr (1992) that GWs are slowed within a superconductor. Although the precise magnitude of the Li and Torr effect is currently controversial, if this effect occurs at all then fabrication and demonstration of this novel component will be possible. Therefore, in this sense, this component may also be regarded as a possible test of the $\mathrm{Li}$ and Torr result, as an alternative or in addition to simply measuring the GW velocity directly through a superconductor.

This lens may be combined, in the manner well-established in conventional optics, with other fixed (or variable) focussing arrangements to produce versatile variable-focus VHFGW components. Technologically useful values of focal length are readily achievable using design parameters that would be typical for a designer having superconductors and electrical coils readily available.

This component is unparalleled in conventional optics because the focal length is varied completely electrically, with no mechanical moving parts. Since there are no moving parts the focal length may be controlled entirely electrically with much greater reliability than could be made by copying equivalent optical glass lens designs. This control can also be considerably faster, since the maximum speed of the electrical control is limited only by electronic circuit design, whereas the speed of conventional moving-optics designs is determined by the limited speeds of mechanical actuators and motors. Therefore, fast auto-focusing, zooming, and imaging tomography using electronic servos may become possible as a result of the development of the necessary VHFGW detectors.

\section{NOMENCLATURE}

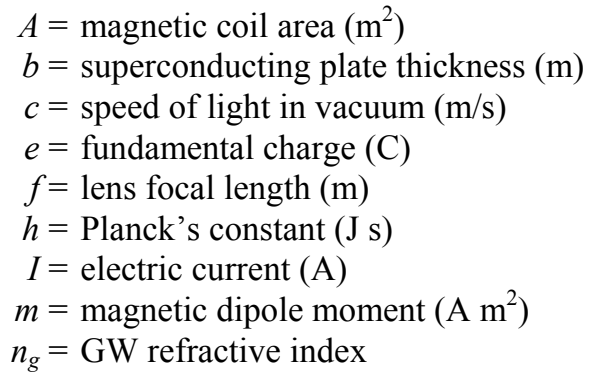

$$
\begin{aligned}
r & =\text { radius in cylindrical coordinates }(\mathrm{m}) \\
R & =\text { radius in spherical polar coordinates }(\mathrm{m}) \\
s & =\text { distance from coil to superconductor }(\mathrm{m}) \\
\theta & =\text { angle in spherical polar coordinates }(\mathrm{rad}) \\
\lambda_{\mathrm{o}} & =\text { VHFGW wavelength in free space }(\mathrm{m}) \\
\mu_{\mathrm{o}} & =\text { permeability of free space }(\mathrm{H} / \mathrm{m}) \\
\phi & =\text { VHFGW phase shift }(\mathrm{rad}) \\
\Phi_{\mathrm{o}} & =\text { magnetic flux through vortex }(\mathrm{Wb})
\end{aligned}
$$

\section{REFERENCES}

Cheng, D.K., Field and Wave Electromagnetics, 2nd. Ed., Addison-Wesley, Reading, MA, 1989, p. 240

Douglass D.H. and Braginsky, B., "Gravitational-Radiation Experiments," in "General relativity: an Einstein centenary survey" edited by S.W. Hawking and W. Israel, CUP, 1979, pp. 90-137.

Einstein, A., Näherungsweise integration der feldgleichungen der gravitation, Sitzungsberichte der Königlich Preußischen Akademie der Wissenschaften, Berlin, 1916, pp. 688-696. 
Forward, R.L. and Miller, L.R., "Generation and Detection of Dynamic Gravitational-Gradient Fields,” J. Appl. Phys. 38 512518 (1967).

Li, N. and Torr, D.G., "Gravitational Effects on the Magnetic Attenuation of Superconductors," Phys. Rev. B 46, 5489-5495 (1992).

Romero, F.B. and Dehnen, H., "Generation of Gravitational Radiation in the Laboratory,” Z. Naturforsch. A 36 948-955 (1981).

Taylor, J.H., "Binary Pulsars and Relativistic Gravity,” Rev. Modern Phys. 66 711-719 (1994).

Torr, D.G. and Li, N., "Gravitoelectric-electric coupling via superconductivity," Found. Phys. Letts. 6, 371-383 (1993).

Woods, R.C. and Baker, R.M.L. "Gravitational Wave Generation and Detection Using Acoustic Resonators and Coupled Resonance Chambers," in Proceedings of Space Technology and Applications International Forum (STAIF-05), edited by M.S. El-Genk, AIP Conference Proceedings, Melville, NY, 2005.

Woods, R.C., "Manipulation of Gravitational Waves for Communications Applications using Superconductors," Physica C 433 101-107 (2005).

Woods, R.C., "A Novel Variable-Focus Lens for HFGW" in Proceedings of Space Technology and Applications International Forum (STAIF-06), edited by M.S. El-Genk, AIP Conference Proceedings, Melville, NY, 2006a.

Woods, R.C., "Enhanced Gertsenshtein Effect in Type-II Superconductors," Physica C 442 85-90 (2006b). 